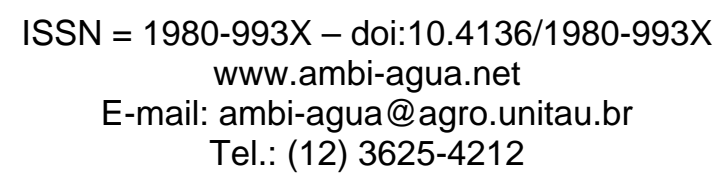

\title{
Cambio en la cobertura y uso de suelo en el norte de Jalisco, México: Un análisis del futuro, en un contexto de cambio climático
}

\author{
(http://dx.doi.org/10.4136/ambi-agua.189) \\ José L. Ibarra-Montoya ${ }^{1,2}$; Rigoberto Román²; Karla Gutiérrez²; \\ Jacobo Gaxiola ${ }^{2}$; Víctor Arias ${ }^{2}$; Maximiano Bautista ${ }^{3}$ \\ ${ }^{1}$ Centro de Investigación y Asistencia en Tecnología y Diseño del Estado de Jalisco, A. C. \\ e-mail: jibarra@ecologia.unam.mx \\ ${ }^{2}$ SAP Servicios Ambientales Profesionales S. C. \\ email: rigoroman@gmail.com; karlationg@hotmail.com; jacobo.sap@gmail.com; victor.sap@gmail.com; \\ ${ }^{3}$ Servicios de Ingeniería de Occidente Tlaloc, S. De R. L. De C.V. \\ e-mail: bautista.maximiano@gmail.com
}

\section{RESUMEN}

En este trabajo se intenta contribuir a mejorar la comprensión de los procesos de cambio en la cobertura y uso de suelo en el tiempo, a nivel regional. Los cambios de cobertura de la vegetación y el uso del suelo se han reconocido en muchos países como una de las principales causas de deterioro ambiental, por ello, están ubicados en el centro de la investigación ambiental y representan un punto importante en diferentes ámbitos como medio para entender los mecanismos de este proceso de deterioro y guía para la toma razonable de decisiones sobre el uso del territorio. La subcuenca Huichol-Atengo ha proveído un conjunto de bienes y servicios a las comunidades que involucra, principalmente a los Wixarikas (comunidades Huicholas); desafortunadamente, esta relación ha repercutido en un deterioro acelerado de sus recursos naturales. En este trabajo se analizan los cambios en la cobertura y uso de suelo en la subcuenca, generando escenarios para los años 1976 y 2000, a partir del empleo de coberturas del suelo por la vegetación y su manejo en un Sistema de Información Geográfica (SIG). Además, se ilustra una metodología, a partir de un modelo de cambio climático que predice un escenario de cambio en la cobertura y uso de suelo para el año 2040. Los resultados muestran que el paisaje de la subcuenca está dominado por el bosque de pino, el bosque de encino y la selva baja caducifolia. La dinámica de cambio está centrada en los tipos de cobertura "bosque de pino" y "bosque de encino", durante el periodo de 1976-2000; la primera disminuye a una tasa anual de $0.20 \%$ y la segunda a $0.76 \%$; y en el periodo estimado de 2000-2040 se revierte este patrón, el bosque de pino disminuye a una tasa anual de $8.95 \%$ y el bosque de encino a 2.11\%. Siendo en ambos casos mayor la pérdida de cobertura vegetal en el segundo periodo analizado. Este patrón de cambio es consistente con las estimaciones reportadas en un contexto de cambio climático.

Palabras clave: deforestación; cambio de uso de suelo; subcuenca Huichol-Atengo; cobertura del terreno.

\section{Change in land cover and land use in the north of Jalisco, Mexico: An analysis of the future in a context of climate change}

\section{ABSTRACT}

This paper focused on the understanding of changes of land cover and land use processes over time, at a regional level. Changes in vegetation cover and land use have been recognized in many countries as a major cause of environmental degradation, therefore, are located in the

Revista Ambiente \& Água - An Interdisciplinary Journal of Applied Science: v. 6, n. 2, 2011. 
IBARRA-MONTOYA, J. L.; ROMAN, R.; GUTIÉRREZ, K.; GAXIOLA, J.; ARIAS, V.; BAUTISTA, M. Cambio en la cobertura y uso de suelo en el norte de Jalisco, México: Un análisis del futuro, en un contexto de cambio climático. Ambi-Agua, Taubaté, v. 6, n. 2, p. 111-128, 2011. (doi:10.4136/ambi-agua. 189)

center of environmental research and represent an important point in different areas as a mean to understand the mechanisms of this process of deterioration and a guide to support decisions on land use policy. The Atengo Huichol sub-basin has provided a set of goods and services to the communities of its surrounds, especially the Wixarikas (Huichol Community). Unfortunately, this relationship has resulted in a rapid deterioration of its natural resources. In this paper, the changes in coverage and land use in the watershed were analyzed, based on scenarios of 1976 and 2000, considering land use and vegetation cover in a Geographic Information System (GIS). In addition, the methodology, based on a model of climate change scenario to predict changes in land cover and land use in 2040 was developed. The results show that the landscape of the watershed is dominated by pine forest, oak forest and tropical deciduous forest. The dynamics of change is centered on the types of coverage "pine" and "oak" forest during the period 1976-2000, the former decreased at an annual rate of $0.20 \%$ and the second at $0.76 \%$ and in the estimated period of $2000-2040$ this pattern is reversed, the pine forest decreased at an annual rate of $8.95 \%$ and oak forest to $2.11 \%$. For both vegetation covers, the loss of vegetation in the second period analyzed was greater. This pattern of change is consistent with estimates previously reported in the context of climate change.

Keywords: deforestation; land use change; watershed Atengo-Huichol; ground cover.

\section{Mudanças no uso da cobertura da terra no norte de Jalisco, México: Uma análise do futuro em um contexto de mudança climática}

Nesta pesquisa foi objetivado melhorar a compreensão dos processos de mudança na cobertura e uso da terra ao longo do tempo, no nível regional. Alterações na cobertura vegetal e uso da terra têm sido reconhecidos, em muitos países, como uma das principais causas da degradação ambiental, portanto, centralizam esforços da pesquisa ambiental e representam um ponto importante em diversas áreas como meio para compreender os mecanismos desse processo de deterioração, fundamental para a tomada de decisões sobre uso da terra. A subbacia Huichol-Atengo tem proporcionado um conjunto de bens e serviços para as comunidades que a envolvem principalmente os Wixarikas (Comunidade dos Huicholas), infelizmente, essa relação resultou em uma rápida deterioração de seus recursos naturais. Neste artigo, as mudanças na cobertura e uso da terra na bacia hidrográfica foram analisadas, pela criação de cenários de 1976 a 2000, baseando-se no uso e na cobertura do solo pela vegetação e de seu manejo em um Sistema de Informação Geográfica (SIG). Além disso, é descrita uma metodologia, baseada em cenários de mudanças climáticas para prever as mudanças na cobertura e uso da terra em 2040. Os resultados mostram que a paisagem da bacia é dominada por floresta de pinheiros, floresta de carvalho e floresta tropical decidual. A dinâmica da mudança é centrada sobre os tipos de cobertura florestal "pinheiro" e "carvalho" durante o período 1976 - 2000. A primeira diminuiu a uma taxa anual de 0,20\% e a segunda, em $0,76 \%$ e, no período estimado de 2000 - 2040, esse padrão é invertido; a floresta de pinheiros decresceu a uma taxa anual de $8,95 \%$ e a floresta de carvalhos para $2,11 \%$. Para ambas as coberturas, a perda da vegetação foi maior no segundo período analisado. Esse padrão de mudança é consistente com estimativas já reportadas no contexto de mudança climática.

Palavras-chave: desmatamento, mudança de uso da terra; sub-bacia Atengo-Huichol; cobertura do solo.

\section{INTRODUCCIÓN}

Los estudios sobre los procesos de cambio en la cobertura y uso del suelo se encuentran en el centro de la atención de la investigación ambiental actual. La mayor parte de los 
IBARRA-MONTOYA, J. L.; ROMAN, R.; GUTIÉRREZ, K.; GAXIOLA, J.; ARIAS, V.; BAUTISTA, M. Cambio en la cobertura y uso de suelo en el norte de Jalisco, México: Un análisis del futuro, en un contexto de cambio climático. Ambi-Agua, Taubaté, v. 6, n. 2, p. 111-128, 2011. (doi:10.4136/ambi-agua. 189)

cambios ocurridos en los ecosistemas terrestres se deben a: i) conversión en la cobertura del terreno; ii) degradación del terreno; e iii) intensificación en el uso del terreno (Lambin, 1997).

Estos procesos, usualmente englobados en lo que se conoce como deforestación o degradación forestal, se asocian a impactos ecológicos importantes en prácticamente todas las escalas. Localmente, inducen la perdida y degradación de suelos, cambios en el microclima y disminución de la diversidad de especies; regionalmente, afectan el funcionamiento de cuencas hidrológicas y de asentamientos humanos, y a nivel global, coadyuvan a las emisiones de gases de efecto invernadero que dan por resultado el problema del cambio climático global.

Por otro lado, el crecimiento exponencial que ha experimentado la población humana a nivel mundial en las últimas décadas, la demanda de recursos naturales para satisfacer las necesidades de supervivencia de esa población y las formas y mecanismos de apropiación de los recursos, con frecuencia ligados a fuerzas sociales, políticas y económicas, han presentado implicaciones estructurales y funcionales sobre los ecosistemas (Mas et al., 2009; Rosete et al., 2009). Estas relaciones hombre-ambiente o entre el ambiente y los procesos ligados a la economía social, se manifiestan o materializan como cambios en la cobertura y uso de suelo (Chen y Yang, 2008) y han sido reconocidos desde hace más de dos décadas como causa importante de cambios en el ambiente global (Turner, 1989).

La deforestación y el cambio en la cobertura y uso del suelo en México, es un problema que se ha presentado desde tiempos precolombinos, sin embargo, durante las últimas cinco décadas este proceso se ha incrementado dramáticamente, con un panorama poco alentador. Según la Organización de las Naciones Unidas para la Agricultura y la Alimentación (FAO), las 52 millones de hectáreas de bosques y selvas con que contaba el país en el año 2000, presentaron una tasa de deforestación promedio de 631 mil ha/año (FAO, 2005).

Esta última cifra puede diferir con la reportada por otras instituciones o investigaciones (Velázquez et al., 2002; México, 2005); sin embargo se ubica entre los rangos extremos reportados para México por la SEMARNAT (México, 2005) (316-800mil ha/año).

Estudios de caso regionales conducidos con diferentes técnicas y metodologías, han reportado tasas de deforestación entre uno y ocho por ciento anual, dependiendo de la región, tipo de vegetación y periodo estudiado (Dirzo y García 1991; Trejo y Hernández, 1996; Mas et al., 1996 y 2009).

Lo anterior, ha situado a los procesos de cambio en la cobertura y uso del suelo en el centro de investigación ambiental actual y representan un punto de importancia en los ámbitos gubernamental, académico y social (García y Mas, 2008), donde el análisis de cambio en la cobertura y uso del suelo representan un medio para entender los mecanismos de este proceso de deterioro y constituye una guía útil para la toma razonable de decisiones sobre el uso del territorio (Chen y Yang, 2008), que en el país y el estado han sido aplicados por diferentes investigadores con fines de monitoreo de hábitat, de monitoreo de áreas de protección incorporadas en la caracterización del paisaje o como información base para instrumentos de planeación como el ordenamiento ecológico, impacto ambiental sectorial y, de manera más reciente relacionadas con el cambio climático como los trabajos de Nájera et al. (2000), Reyes et al. (2006), Márquez (2008), González et al. (2009), Rosete et al. (2009).

La subcuenca Huichol-Atengo, ha proveído de un conjunto de bienes y servicios a los habitantes de las localidades que en ella se asientan; desafortunadamente esta relación carente de una planeación que incorpore criterios ambientales, ha repercutido en un deterioro acelerado de sus recursos naturales, tales como, disminución de superficie de bosques y selvas, alteraciones en la cantidad y calidad de agua, calidad de aire, suelos y posibles implicaciones climáticas, que en el tiempo, se han traducido en bajos niveles de bienestar de los habitantes del territorio.

De esta forma, la subcuenca se proyecta como un campo de investigación que es necesario atender, a fin de demostrar algunos de estos procesos, generar conocimiento que 
IBARRA-MONTOYA, J. L.; ROMAN, R.; GUTIÉRREZ, K.; GAXIOLA, J.; ARIAS, V.; BAUTISTA, M. Cambio en la cobertura y uso de suelo en el norte de Jalisco, México: Un análisis del futuro, en un contexto de cambio climático. Ambi-Agua, Taubaté, v. 6, n. 2, p. 111-128, 2011. (doi:10.4136/ambi-agua. 189)

contribuya a una mejor toma de decisiones y en la planificación de sectores como el urbano, agrícola y forestal, o bien que sirva de base en la integración de instrumentos de planeación del territorio, por lo que el presente trabajo tiene como objetivo evaluar los cambios en la cobertura y uso del terreno en la subcuenca Huichol-Atengo, ubicada en la porción norte del sistema hidrológico Lerma-Santiago, que incluye a los estados de Jalisco, Nayarit, Zacatecas y Durango en los años 1970, 2000 y realizar una proyección hacia el año 2040.

\section{2. Área de Estudio}

La subcuenca Huichol-Atengo se localiza en los estados de Jalisco, Nayarit, Zacatecas y Durango, entre las coordenadas $104^{\circ} 48^{\prime} 19.452^{\prime \prime} \mathrm{E}$; $22^{\circ} 36^{\prime} 28.999^{\prime}$ N N, 104 48' 23.911” E;

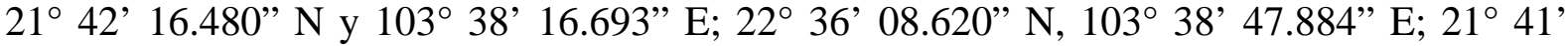
56.998” N (UTM; “X” 520,000; 640,000 y “Y” 2,400,000; 2,500,000) (Figura 1). Con una superficie de 1,180,847.313ha o $11,808.47 \mathrm{Km}^{2}$ (INE, 2000). Forma parte del sistema hidrológico Lerma-Santiago, su área de escurrimiento está delimitada por elevaciones de origen volcánico aisladas, formando un relieve heterogéneo donde es posible el establecimiento de diferentes tipos de vegetación. En las partes altas de las montañas se encuentra dominando la vegetación de bosque de pino, bosque de pino-encino, bosque de encino y en altitudes menores la selva baja caducifolia. Este tipo de selva coincide con la región biogeográfica que incluye la vegetación del pacífico (INE, 2000). Además en las partes más bajas de la subcuenca existe una vegetación de tipo pastizal (Rzedowski, 2005).

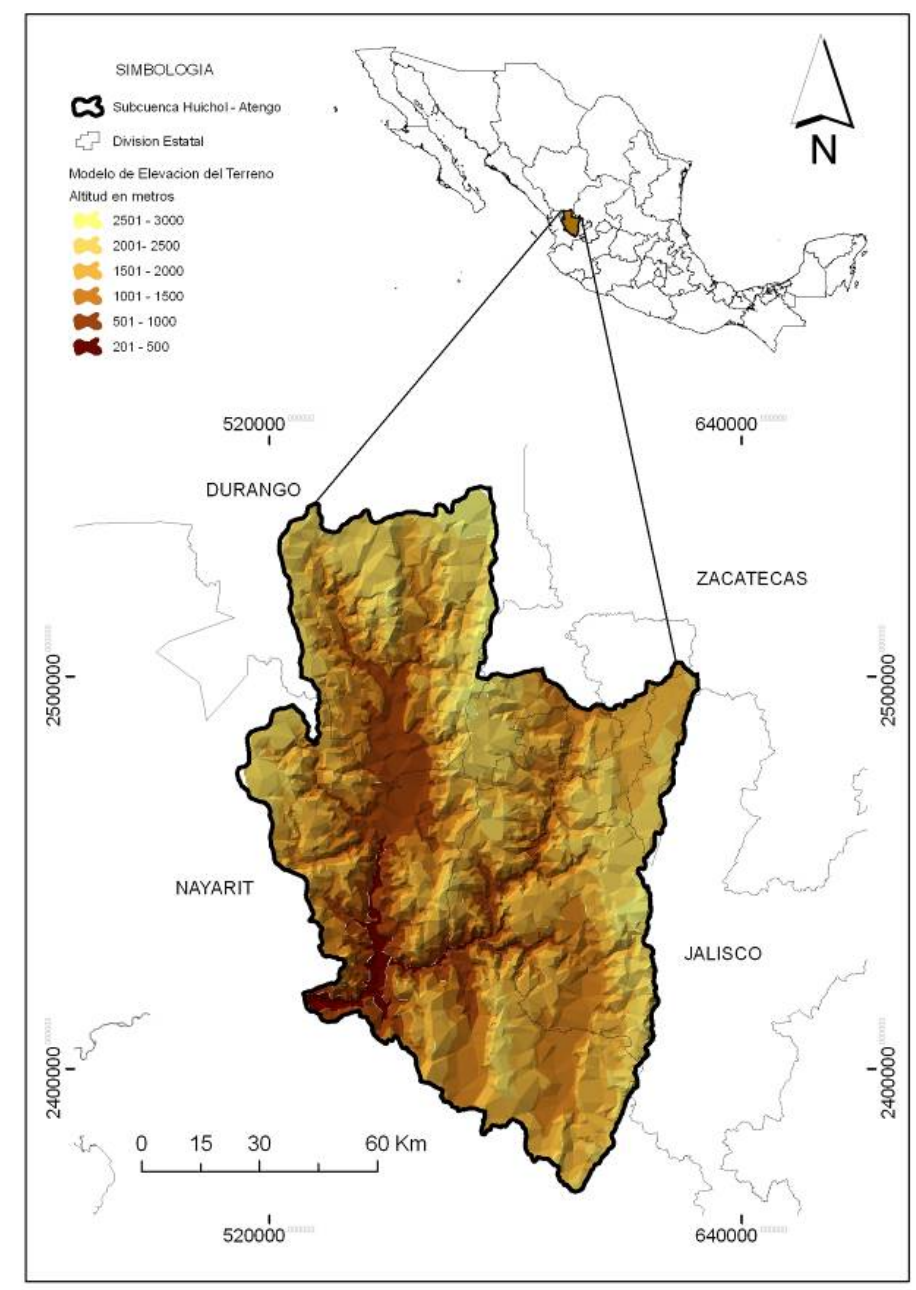

Figura 1. Mapa de localización geográfica, y topografía de la subcuenca Huichol-Atengo. (INE, 2000). 


\section{MATERIALES Y MÉTODOS}

Los cambios en la cobertura y uso de suelo del terreno de la subcuenca se analizaron a partir del uso de un sistema de información geográfica (ArcMap 9.2; GIS, ESRI, 1999-2001), empleando las coberturas en formato vectorial de los años 1976 y 2000, a una escala de 1:250,000; dichas coberturas se encuentran disponibles en el portal "Espacio Digital Geográfico de la Secretaria del Medio Ambiente y Recursos Naturales (SEMARNAT) (INE, 2000). La metodología que se llevó a cabo para predecir el escenario de cobertura de uso de suelo y vegetación del año 2040 se menciona posteriormente.

La definición de la cobertura vegetal adoptó la clasificación de Rzedowski (2005). Mientras que los usos asociados a cada cobertura fueron identificados por las actividades económicas de la región: i) vida silvestre y forestal; ii) vida silvestre y agrícola; iii) agrícola y forestal; iv) agrícola; v) pecuario; vi) habitacional; y vii) agropecuario.

El análisis comparativo de los años 1976, 2000 y 2040, se realizó con los datos de superficie por cada tipo de cobertura, en formato digital y bajo la misma proyección geográfica. Se calcularon las tasas de cambio de "vegetación natural”, de bosques y selvas, así como de los pastizales tanto inducidos como naturales, mediante un modelo exponencial discreto sugerido por Trejo y Dirzo (2000):

$$
r=1-\left(1-\frac{A 1-A 2}{A 1}\right) \wedge 1 / t
$$

donde:

$r$, es la tasa de cambio anual

$\mathrm{A}_{1}$, es el área cubierta por vegetación al inicio del periodo

$A_{2}$, es el área cubierta por vegetación al final del periodo

t, es el número de años del periodo de análisis

\subsection{Desarrollo del modelo para predecir el uso de suelo del año 2040 en los estados de Jalisco, Nayarit, Durango y Zacatecas.}

Para modelar el comportamiento de la vegetación del año 2040 de la subcuenca HuicholAtengo, se tomaron en cuenta los estados de Jalisco, Nayarit, Durango y Zacatecas; y se utilizaron las variables de temperatura y humedad usadas en el trabajo de Duran (2010).

Procedimiento

i) Se empleó el modelo de sensibilidad propuesto por Villers y Trejo (2010), según este modelo, los tipos de vegetación más afectados por las variaciones climáticas son los que están expuestos a condiciones más secas y más cálidas, señaladas como menor humedad $\left(-10 \%\right.$ en la precipitación) y mayor temperatura $\left(+2{ }^{\circ} \mathrm{C}\right)$.

ii) Con ayuda del Arc Map 9.2, se realizó una búsqueda de las áreas geográficas que presentaran estas dos condiciones (aumento de temperatura y disminución de humedad) y se realizó una sumatoria de "raster", en el módulo de estadística geográfica.

iii) Para conocer los tipos de vegetación actual que podrían verse afectados por el cambio de temperatura y humedad, se utilizó el mapa de uso de suelo y vegetación, escala 1:250,000, del Instituto Nacional de Ecología-INE (INE, 2000). Sobre este mapa y utilizando el software de sistemas de información geográfica conocido como Kosmo versión 2.0, se sobrepusieron los mapas de zonas con aumento significativo de temperatura y reducción de la precipitación. 
iv) Se obtuvo un mapa con la predicción de uso de suelo y vegetación para el año 2040, y se editó en ArcMap 9.2. (GIS, ESRI, 1999-2001).

\section{RESULTADOS Y DISCUSIÓN}

\subsection{Modelo para predecir un escenario para el año 2040 en un contexto de cambio climático de los estados de Jalisco, Nayarit, Durango y Zacatecas}

Según el modelo de sensibilidad propuesto por Villers y Trejo (2010), los tipos de vegetación más afectados por las variaciones climáticas corresponderían al bosque mesófilo de montaña, seguido de los bosques templados, el bosque espinoso y el bosque tropical caducifolio. Las zonas más sensibles en las cuales se ubican los bosques templados son: la Sierra Madre occidental, en los estados de Chihuahua y Durango, norte de Jalisco, y las serranías al sur de Chapala.

Para analizar el posible efecto del cambio climático en el área de estudio, se tomaron como insumo los modelos de comportamiento de la temperatura y la humedad elaborados por (Durán, 2010) para el año 2040. Según el modelo de Durán (2010), las zonas con mayor cambio de temperatura se ubican en la vertiente del pacífico, los mayores cambios se reflejan al sur de Durango y Norte del estado de Nayarit. La costa del estado de Jalisco registrará cambios en la temperatura cercanos a 1.3 grados (Figura 2).

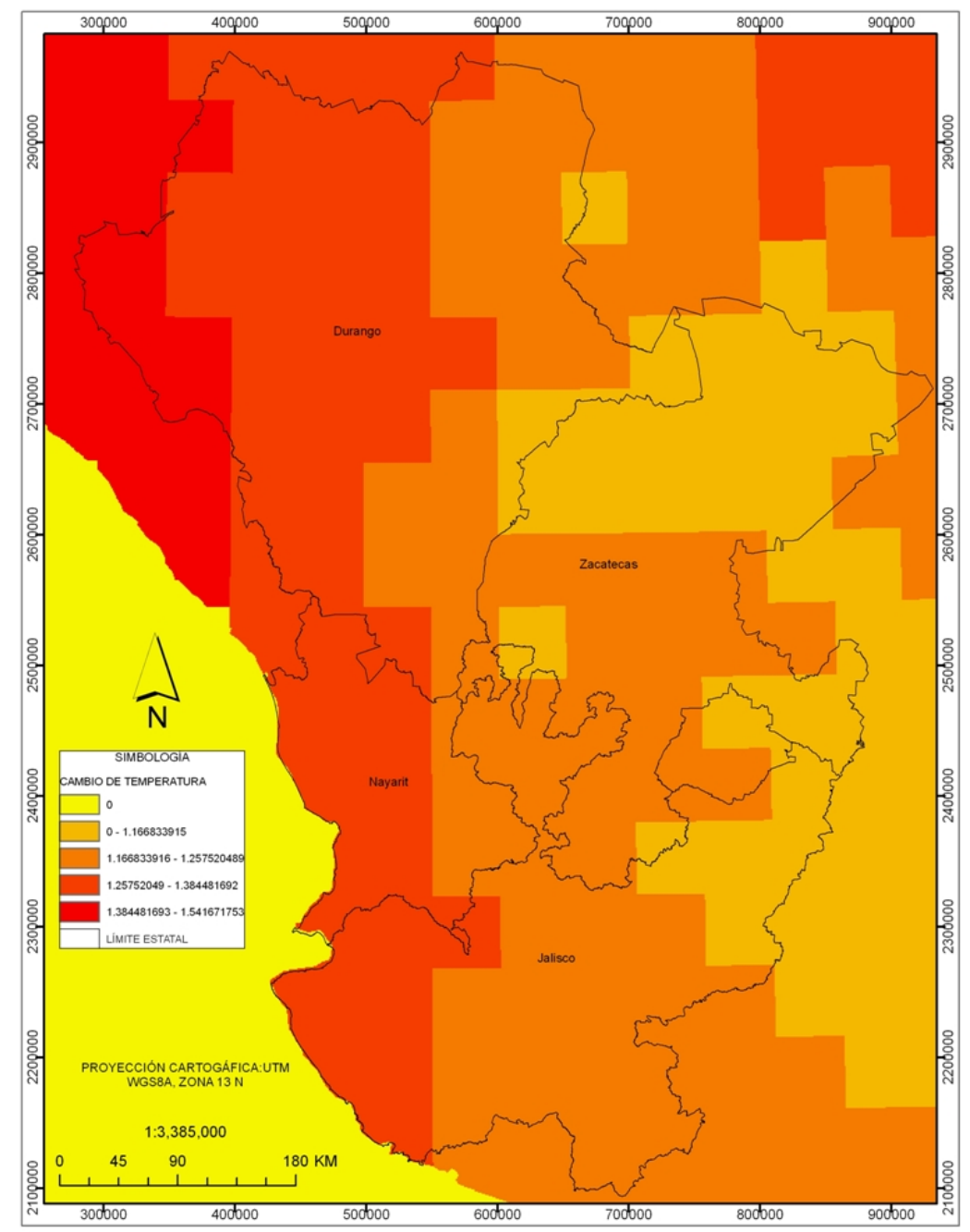

Figura 2. Zonas con incremento significativo de la temperatura. Según Durán (2010).

En cuanto a los cambios en la precipitación, según el modelo planteado por Durán (2010), la disminución de la misma cubre casi todo el estado de Jalisco y completamente el 
IBARRA-MONTOYA, J. L.; ROMAN, R.; GUTIÉRREZ, K.; GAXIOLA, J.; ARIAS, V.; BAUTISTA, M. Cambio en la cobertura y uso de suelo en el norte de Jalisco, México: Un análisis del futuro, en un contexto de cambio climático. Ambi-Agua, Taubaté, v. 6, n. 2, p. 111-128, 2011. (doi:10.4136/ambi-agua. 189)

estado de Nayarit. Los estados menos vulnerables por este cambio son Durango y Zacatecas. En la parte central de la costa de Jalisco se registran disminuciones cercanas a 127ml.

En esa región representan en el año 2010 precipitaciones cercanas a los 1200ml anuales. Esta referencia implica disminuciones del 10\% de precipitación (Figura 3).

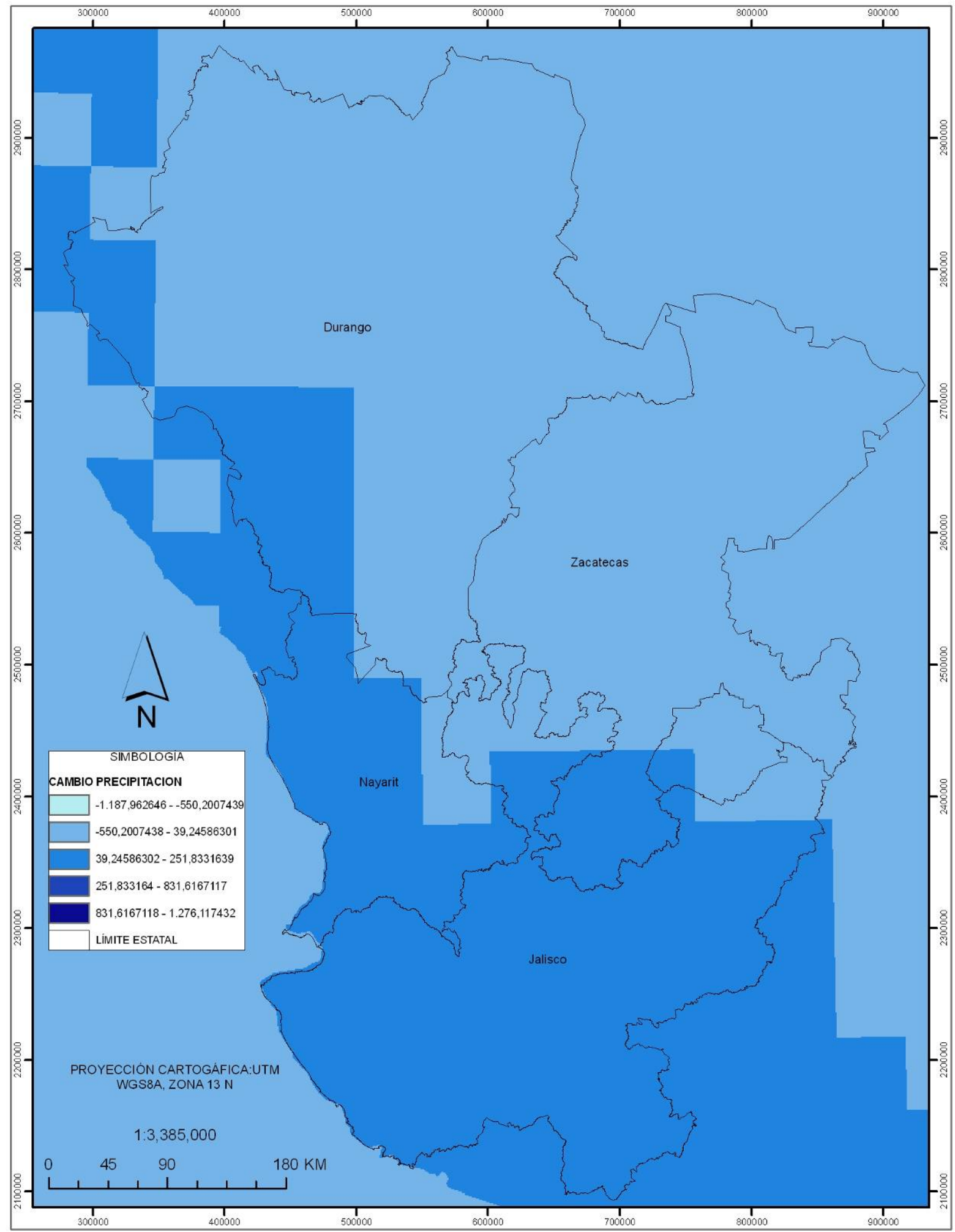

Figura 3. Zonas de disminución significativa de la precipitación (Duran, 2010).

La Costa norte de Jalisco y la parte central de Nayarit que recibe hasta $1400 \mathrm{ml}$ de precipitación anual, tendrían reducciones de hasta 137ml, muy cercano al 10\%. 
IBARRA-MONTOYA, J. L.; ROMAN, R.; GUTIÉRREZ, K.; GAXIOLA, J.; ARIAS, V.; BAUTISTA, M. Cambio en la cobertura y uso de suelo en el norte de Jalisco, México: Un análisis del futuro, en un contexto de cambio climático. Ambi-Agua, Taubaté, v. 6, n. 2, p. 111-128, 2011. (doi:10.4136/ambi-agua. 189)

Sumando estas dos variables, (precipitación y temperatura) resulta que las áreas afectadas serían la costa y Sierra Occidental del estado de Jalisco, la zona norte del estado de Nayarit y en el estado de Durango, toda la vertiente del Pacífico (Figura 4).

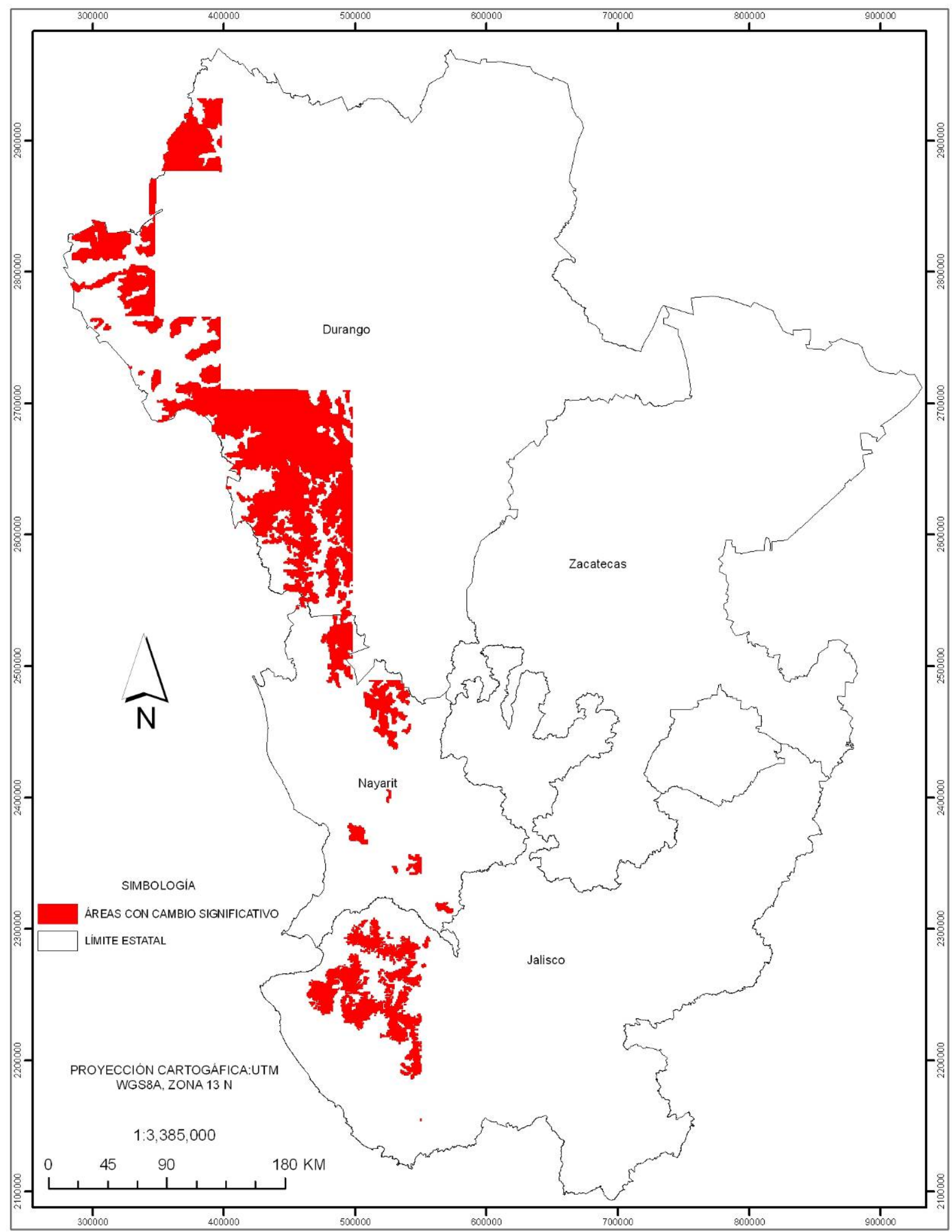

Figura 4. Zonas con un aumento significativo de temperatura y una reducción de la precipitación.

Para conocer los tipos de vegetación actual que se podrían ver afectados por el cambio de temperatura y humedad, se utilizó el mapa de uso de suelo y vegetación del INE (2000). Sobre este mapa y utilizando el software de Sistemas de Información Geográfica (SIG) 
IBARRA-MONTOYA, J. L.; ROMAN, R.; GUTIÉRREZ, K.; GAXIOLA, J.; ARIAS, V.; BAUTISTA, M. Cambio en la cobertura y uso de suelo en el norte de Jalisco, México: Un análisis del futuro, en un contexto de cambio climático. Ambi-Agua, Taubaté, v. 6, n. 2, p. 111-128, 2011. (doi:10.4136/ambi-agua. 189)

conocido como KOSMO versión 2.0, se sobrepusieron los mapas de las zonas de aumento de temperatura y reducción de precipitación (Figura 5).

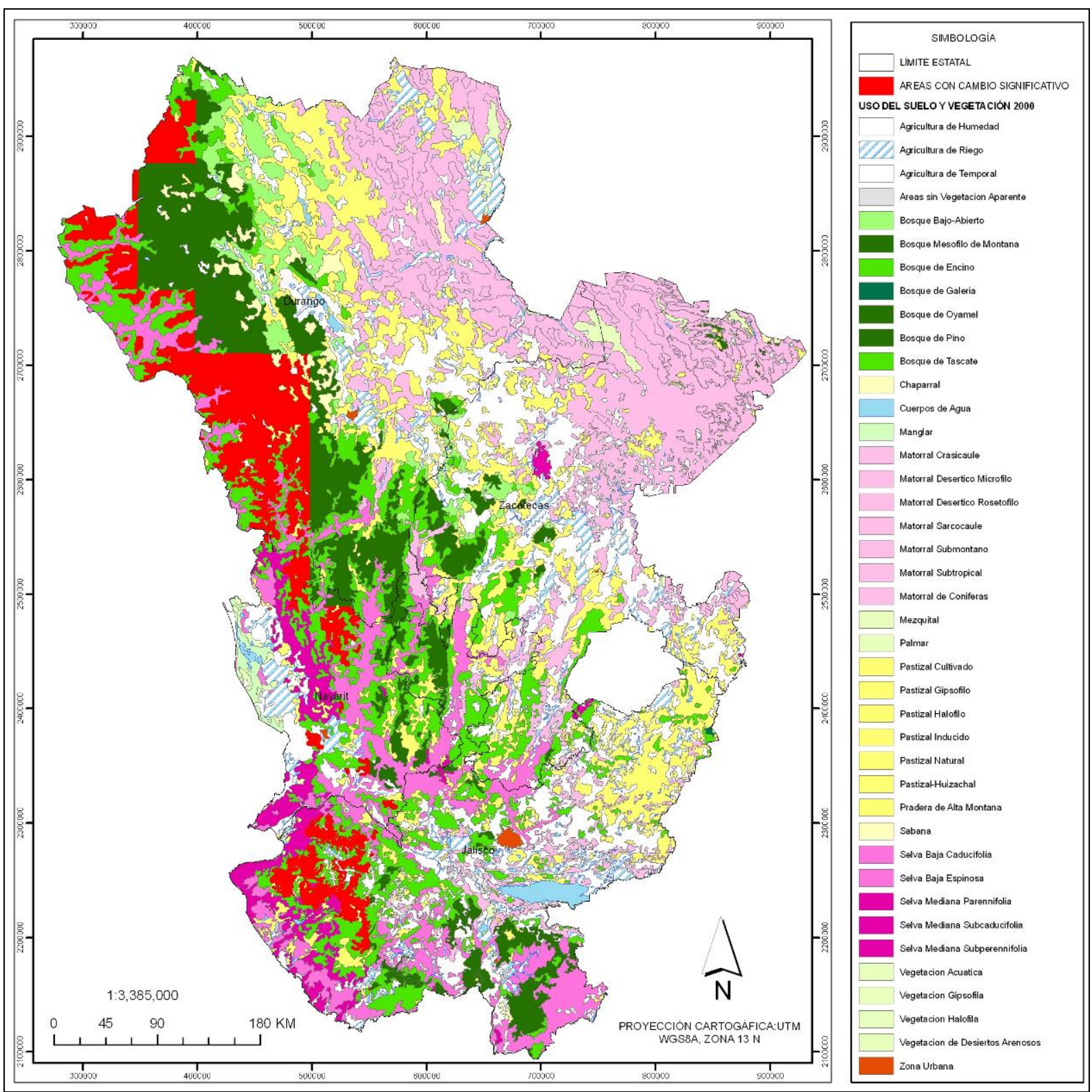

Figura 5. Sobreposición en el SIG del mapa de uso de suelo y vegetación con las zonas con aumento significativo de temperatura y reducción de precipitación.

En los estados de Jalisco los bosques con alto riesgo se ubican en los municipios de Cabo Corrientes, Tomatlán, Villa Purificación, Ayutla, Cuautla, Atenguillo, Puerto Vallarta, San Sebastián del Oeste, Talpa, Guachinango y Mascota. Mientras en el estado de Nayarit se podría ver afectada la vegetación de los municipios de SantaMaría del Oro, Xalisco, Tepic, del Nayar y Huajicori, todas estos municipios ubicados en la subcuenca Huichol-Atengo.

Con este modelo de análisis se calcula que 1,081,127ha de bosque de pino, de los estados de Jalisco, Durango, Nayarit y Zacatecas, estarán en riesgo de transformarse principalmente a bosque de encino ya que esta última comunidad vegetal presenta mayor tolerancia a la disminución de la precipitación y al aumento de la temperatura.

Con la predicción del escenario de uso de suelo y vegetación para el año 2040 de los estados de Jalisco, Nayarit, Durango y Zacatecas (Figura 6), fue posible extraer el mapa para la subcuenca Huichol-Atengo analizada en este trabajo. 
IBARRA-MONTOYA, J. L.; ROMAN, R.; GUTIÉRREZ, K.; GAXIOLA, J.; ARIAS, V.; BAUTISTA, M. Cambio en la cobertura y uso de suelo en el norte de Jalisco, México: Un análisis del futuro, en un contexto de cambio climático. Ambi-Agua, Taubaté, v. 6, n. 2, p. 111-128, 2011. (doi:10.4136/ambi-agua. 189)

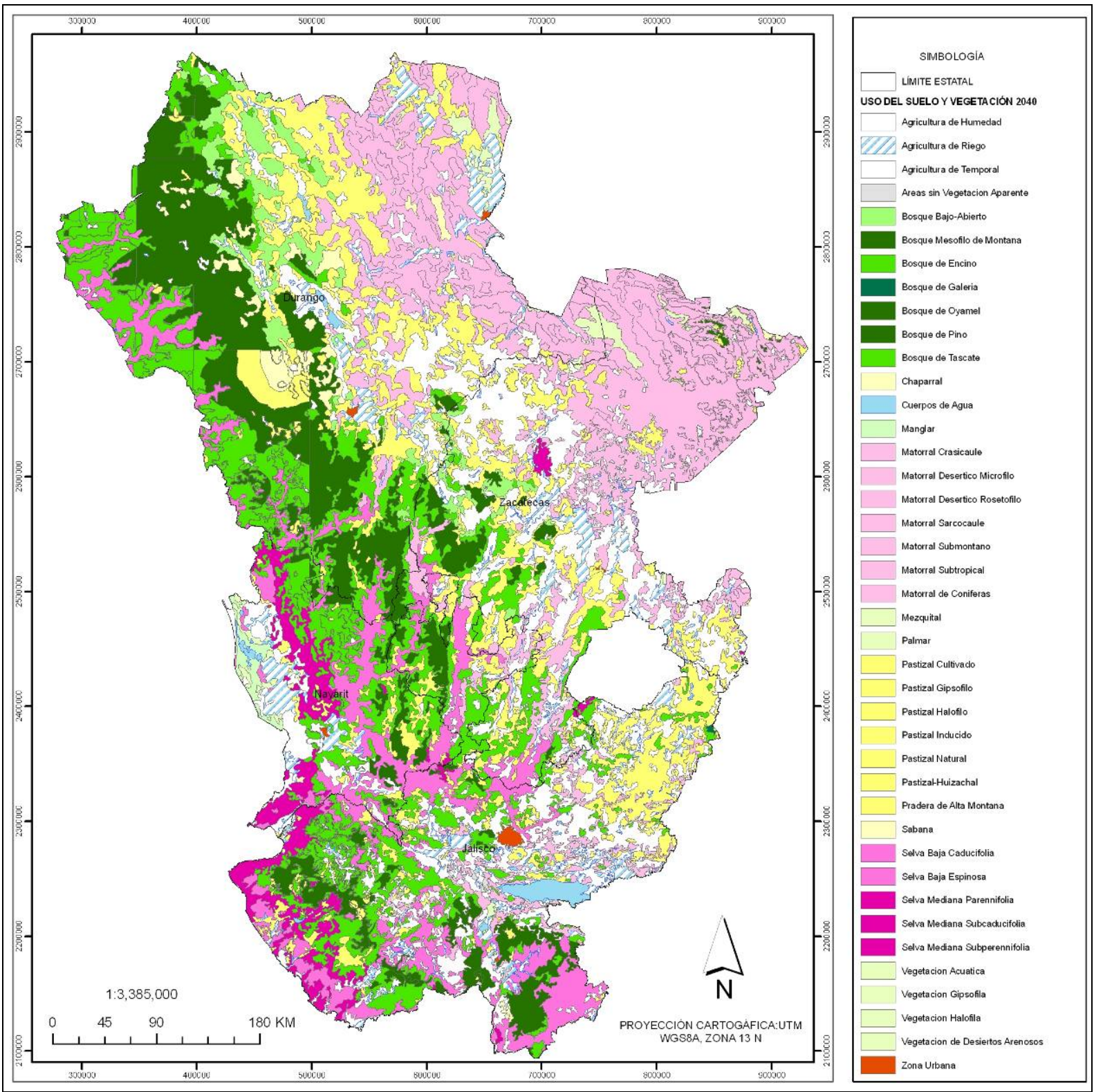

Figura 6. Mapa resultante del uso de suelo y vegetación para el año 2040 por efecto del cambio climático.

\subsection{Cambio en la cobertura y uso del suelo de la subcuenca Huichol-Atengo}

Los resultados del análisis de cambio en la cobertura y uso del suelo para 1976, 2000 y 2040 de la subcuenca Huichol-Atengo, muestran un mosaico compuesto de diez grandes grupos de cobertura, y cinco usos diferentes del suelo (Tabla 1, Figuras 7, 8 y 9), los cuales presentan cambios (pérdidas y ganancias) entre cada periodo analizado.

\subsection{Cambio en la cobertura y uso del suelo de 1976-2000}

Los tipos de cobertura que se han afectado y disminuido en su representación espacial en la subcuenca son el "bosque de pino", "bosque de encino" y "selva baja caducifolia", perdiendo anualmente 25.540, 1,636.132 y 645.079ha respectivamente, resulta evidente que la mayor presión se ha ejercido sobre las asociaciones del bosque de encino, las cuales reportan un cambio neto anual de $0.76 \%$ (Tabla 2, Figuras 7 y 8 ).

En contra parte, los "pastizales inducidos" y el "matorral subtropical” incrementaron su superficie ganando anualmente 3,302.600 y 168.548ha respectivamente, al mismo tiempo los “asentamientos humanos", incrementaron anualmente 5.573ha (Tabla 2). 
IBARRA-MONTOYA, J. L.; ROMAN, R.; GUTIÉRREZ, K.; GAXIOLA, J.; ARIAS, V.; BAUTISTA, M. Cambio en la cobertura y uso de suelo en el norte de Jalisco, México: Un análisis del futuro, en un contexto de cambio climático. Ambi-Agua, Taubaté, v. 6, n. 2, p. 111-128, 2011. (doi:10.4136/ambi-agua. 189)

Tabla 1. Tipos de vegetación y usos del suelo, así como el cambio de cobertura vegetal para los años 1976 y 2000 (INE, 2000). Los datos que se presentan para el año 2040, se obtuvieron a partir del escenario propuesto en el presente trabajo.

\begin{tabular}{|c|c|c|c|c|}
\hline \multirow[t]{2}{*}{ Tipo de vegetación } & \multirow[t]{2}{*}{ Uso de suelo } & \multicolumn{3}{|c|}{ Cobertura (ha) } \\
\hline & & 1976 & 2000 & 2040 \\
\hline $\begin{array}{l}\text { Agricultura de riego (incluye } \\
\text { riego eventual) }\end{array}$ & Agrícola & 167,4 & 1083,2 & ------- \\
\hline Agricultura de temporal & Agrícola & 11602,4 & 25835,2 & 6220,8 \\
\hline Asentamiento humano & Habitacional & 132,6 & 266,3 & ------------ \\
\hline Bosque de encino & $\begin{array}{l}\text { Forestal y vida } \\
\text { silvestre }\end{array}$ & 235362,0 & 196094,9 & 452138,4 \\
\hline Bosque de pino & $\begin{array}{l}\text { Forestal y vida } \\
\text { silvestre }\end{array}$ & 12798,7 & 12186,6 & 3755492,6 \\
\hline $\begin{array}{l}\text { Bosque de pino-encino } \\
\text { (incluye encino-pino) }\end{array}$ & $\begin{array}{l}\text { Forestal, vida silvestre } \\
\text { y agrícola }\end{array}$ & 561867,2 & 517271,9 & ------------ \\
\hline Matorral subtropical & $\begin{array}{l}\text { Pecuario y vida } \\
\text { silvestre }\end{array}$ & 9902,4 & 13947,6 & 10486,7 \\
\hline Pastizal inducido & $\begin{array}{l}\text { Pecuario y vida } \\
\text { silvestre }\end{array}$ & 141201,7 & 220464,1 & 118840,3 \\
\hline $\begin{array}{l}\text { Pastizal natural (incluye } \\
\text { pastizal-huizachal) }\end{array}$ & $\begin{array}{l}\text { Pecuario y vida } \\
\text { silvestre }\end{array}$ & 12716,2 & 12243,1 & ------------ \\
\hline $\begin{array}{l}\text { Selva baja caducifolia y } \\
\text { subcaducifolia }\end{array}$ & $\begin{array}{l}\text { Forestal y vida } \\
\text { silvestre }\end{array}$ & 196936,3 & 181454,4 & 219690,2 \\
\hline
\end{tabular}

Estos datos muestran una dinámica típica de este tipo de coberturas, las cuales son consideradas como principales causas de cambio de cobertura (Turner, 1989). Las tierras desnudas "pastizales inducidos" también tienen la tendencia de incrementarse, en la Tabla 2 se puede observar que para el periodo 1976-2000 hubo un incremento anual espacial de su representación en la subcuenca en $1.87 \%$.

\subsection{Escenario de cambio en la cobertura para $2000-2040$}

Los resultados del análisis de cambio en la cobertura y uso del suelo para la predicción del periodo 2000-2040 de la subcuenca Huichol-Atengo, muestra un mosaico compuesto de seis grandes grupos de cobertura, y tres usos diferentes del suelo (Tabla 1, Figura 9), los cuales presentan cambios (pérdidas y ganancias) entre cada año analizado.

Los tipos de cobertura que se han afectado y disminuido su representación espacial en la subcuenca para el escenario predicho son "bosque de pino", "bosque de encino" y "selva baja caducifolia”, perdiendo anualmente 9,084.07, 6,401.09 y 955.89ha respectivamente, resulta evidente que la mayor presión se ejercerá sobre las asociaciones del bosque de pino, las cuales presentan un cambio neto de $8.95 \%$ (Tabla 2).

En contra parte, los "pastizales" y el "matorral subtropical” incrementaron anualmente su superficie en 2,540.59 y 86.52ha respectivamente (Tabla 2).

Estos datos también muestran una dinámica típica de este tipo de coberturas (Turner, 1989). 
IBARRA-MONTOYA, J. L.; ROMAN, R.; GUTIÉRREZ, K.; GAXIOLA, J.; ARIAS, V.; BAUTISTA, M. Cambio en la cobertura y uso de suelo en el norte de Jalisco, México: Un análisis del futuro, en un contexto de cambio climático. Ambi-Agua, Taubaté, v. 6, n. 2, p. 111-128, 2011. (doi:10.4136/ambi-agua. 189)

Tabla 2. Tasa de cambio anual de la cobertura vegetal para los periodos de tiempo 1976-2000 y 2000-2040.

\begin{tabular}{l|c|c|c|c}
\hline Tipo de vegetación & $\mathbf{1 9 7 6 - 2 0 0 0 ( h a )}$ & $\mathbf{\%}$ & $\mathbf{2 0 0 0 - 2 0 4 0 ( h a )}$ & $\mathbf{\%}$ \\
\hline Agricultura de riego & 38159,0 & 8,1 & -------- & ----- \\
Agricultura de temporal & 593034,0 & 3,4 & $-490,4$ & 3,5 \\
Asentamiento humano & 5573,0 & 3,0 & -------- & ------ \\
Bosque de encino & $-1636132,0$ & 0,8 & 6401,1 & 2,1 \\
Bosque de pino & $-25504,0$ & 0,2 & 9084,1 & 9,0 \\
Bosque de pino-encino & $-1858138,0$ & 0,3 & --------- & ------ \\
Matorral subtropical & 168548,0 & 1,4 & $-86,5$ & 0,7 \\
Pastizal inducido & 3302600,0 & 1,9 & $-2540,6$ & 1,5 \\
Pastizal natural & $-19712,0$ & 0,2 & -------- & ----- \\
Selva Baja Caducifolia & $-645079,0$ & 0,3 & 955,9 & 0,5 \\
\hline
\end{tabular}

\subsection{Tasa de deforestación para 1976-2000}

La tasa anual de deforestación calculada para el periodo 1976-2000, de la vegetación de "bosque de encino" y "bosque de pino" fue de 0.76 y $0.20 \%$, la cual es similar a la tasa nacional y la estatal reportada por la UNAM para el período 1993-2000 y CONAFORSEMARNAT para 1990-2000 que es de 0.57\% y 1.14\% respectivamente (México, 2006), así como las obtenidas por Nájera et al. (2000) de 0.44\% (1970-1986), 2.35\% (1986-1995), para la Sierra de San Juan.

La tasa de deforestación anual de los bosques de encino y de pino es de $1.3 \%$, mientras que la selva baja caducifolia presenta una tasa de $0.34 \%$. Ambos resultados son similares a los reportados por SEMARNAT (México, 2006) para estas asociaciones en el estado de Nayarit y las reportadas para Chiapas por Ochoa y González (2000) y San Luís Potosí por Reyes et al. (2006), quienes reportan tasas entre 1.1 y 3.4\% y 2.0 y 11\%, respectivamente.

\subsection{Tasa de deforestación para 2000-2040}

La tasa anual de deforestación calculada para el periodo de 2000-2040, de la vegetación de "bosque de encino" y "bosque de pino" es de 2.11 y 8.95\%. La tasa anual de deforestación de los bosques de encino y de pino es de $11.06 \%$, mientras que la selva baja caducifolia presenta una tasa anual de $0.48 \%$. Ambos resultados son similares a los reportados por SEMARNAT (México, 2006) para estas asociaciones en el estado de Nayarit y Jalisco.

El análisis del cambio de cobertura del período 1976-2040, revela un paisaje dominado principalmente por tierras de cultivo sobre todo en las partes bajas de la subcuenca, el bosque de encino y de pino y la selva baja caducifolia. Sin embargo, la dinámica de cambio de cobertura y uso del suelo se concentra en la vegetación de pino y encino, de hecho el cambio es de encino por el pino, como se puede observar en la cobertura espacial; el encino aumenta su cobertura vegetal ocupando las áreas que correspondían a la vegetación de pino. Este fenómeno se observa mucho mejor en el año 2040 (Figura 9). 
IBARRA-MONTOYA, J. L.; ROMAN, R.; GUTIÉRREZ, K.; GAXIOLA, J.; ARIAS, V.; BAUTISTA, M. Cambio en la cobertura y uso de suelo en el norte de Jalisco, México: Un análisis del futuro, en un contexto de cambio climático. Ambi-Agua, Taubaté, v. 6, n. 2, p. 111-128, 2011. (doi:10.4136/ambi-agua. 189)

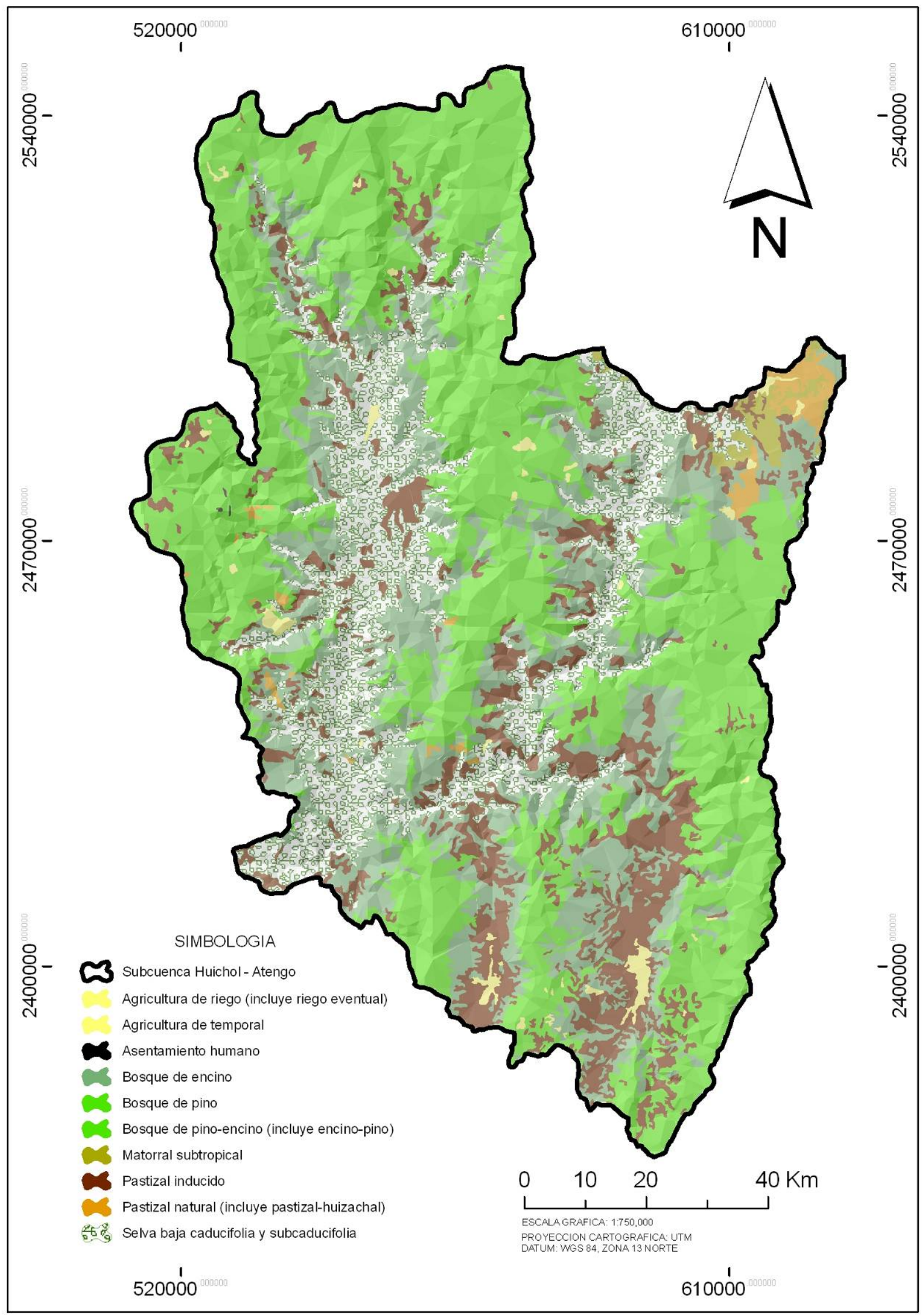

Figura 7. Cobertura del terreno de la subcuenca Huichol-Atengo en 1976. 
IBARRA-MONTOYA, J. L.; ROMAN, R.; GUTIÉRREZ, K.; GAXIOLA, J.; ARIAS, V.; BAUTISTA, M. Cambio en la cobertura y uso de suelo en el norte de Jalisco, México: Un análisis del futuro, en un contexto de cambio climático. Ambi-Agua, Taubaté, v. 6, n. 2, p. 111-128, 2011. (doi:10.4136/ambi-agua. 189)

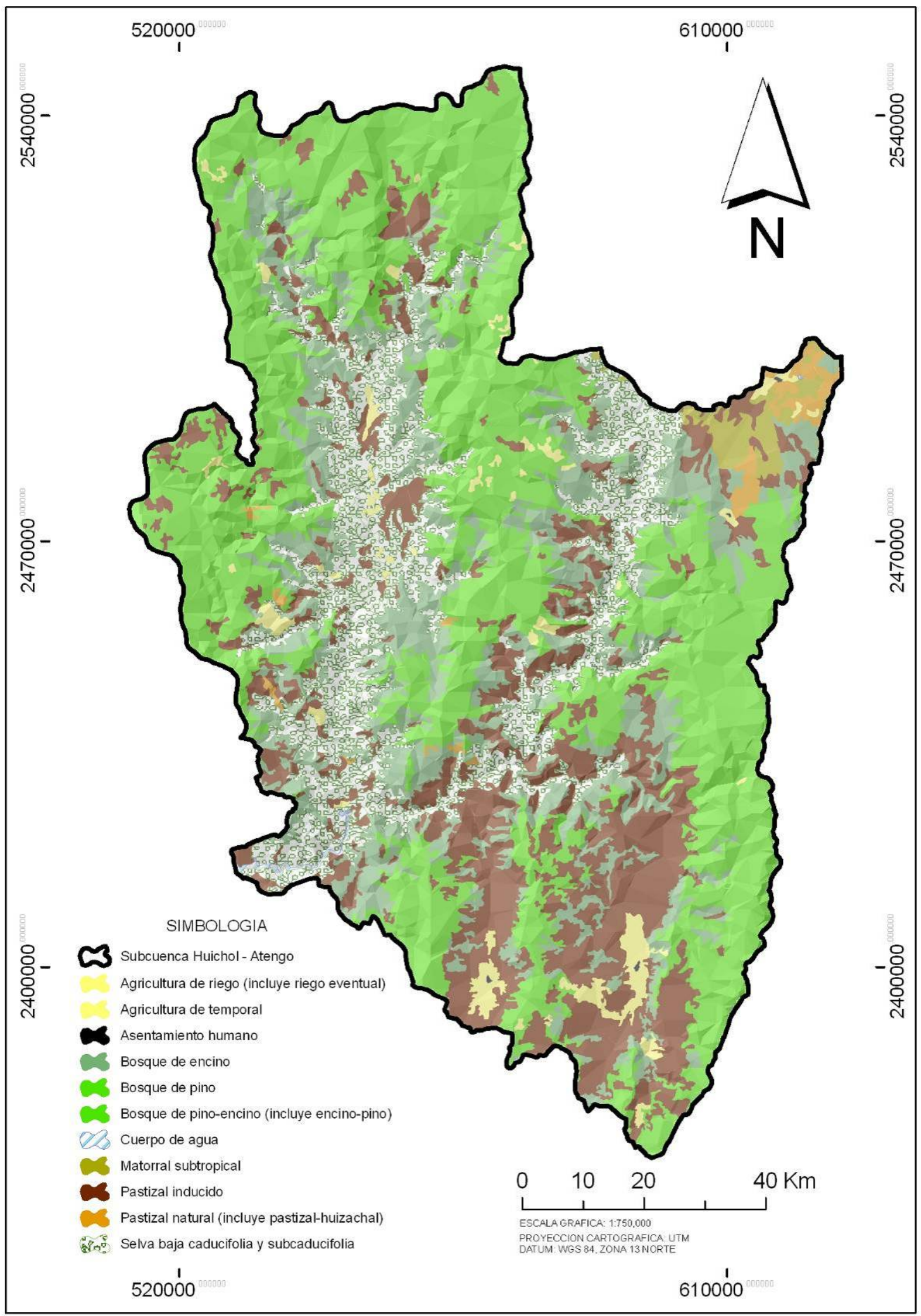

Figura 8. Cobertura del terreno de la subcuenca Huichol-Atengo en 2000. 
IBARRA-MONTOYA, J. L.; ROMAN, R.; GUTIÉRREZ, K.; GAXIOLA, J.; ARIAS, V.; BAUTISTA, M. Cambio en la cobertura y uso de suelo en el norte de Jalisco, México: Un análisis del futuro, en un contexto de cambio climático. Ambi-Agua, Taubaté, v. 6, n. 2, p. 111-128, 2011. (doi:10.4136/ambi-agua. 189)

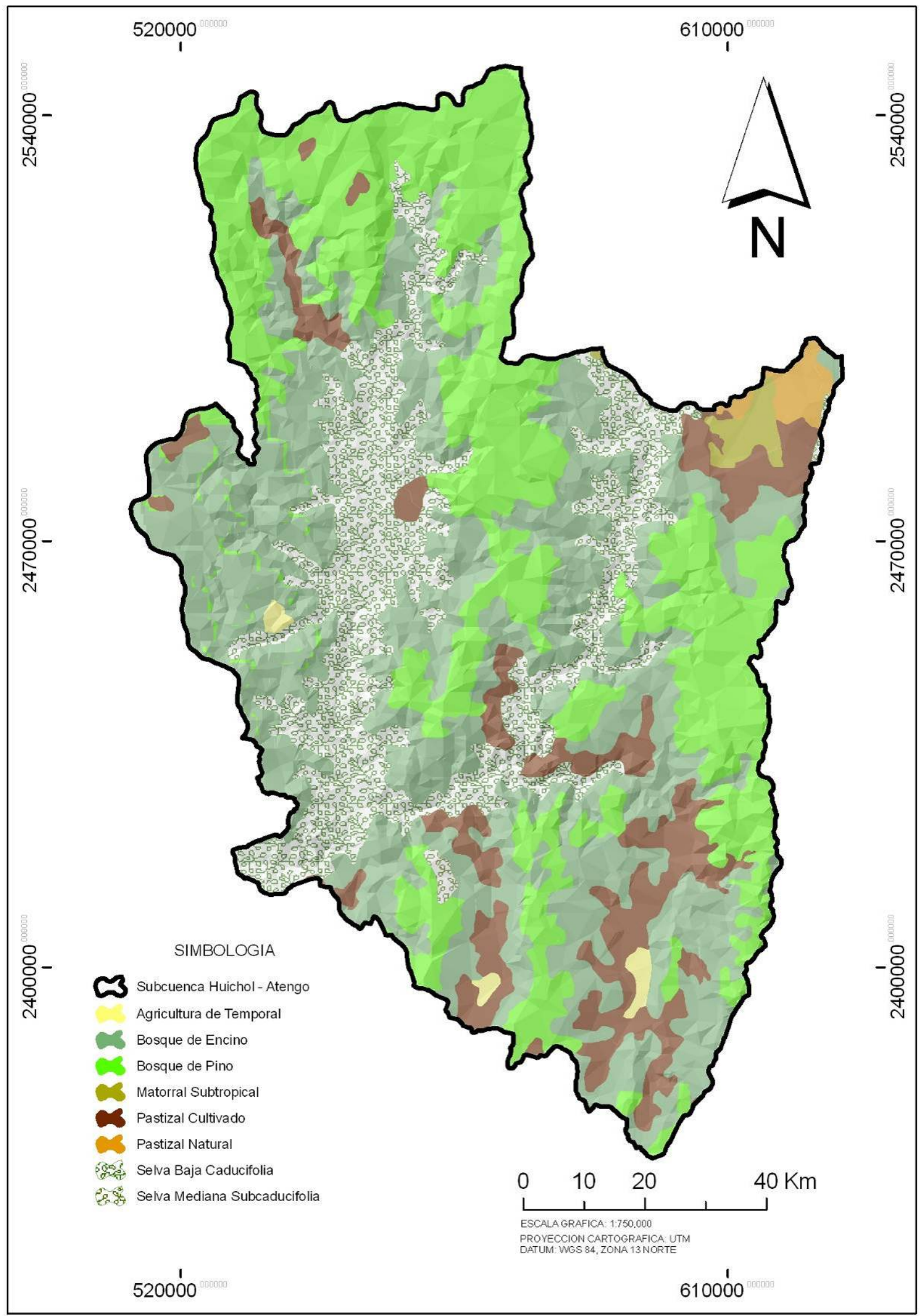

Figura 9. Cobertura del terreno de la subcuenca Huichol-Atengo en 2040. 
IBARRA-MONTOYA, J. L.; ROMAN, R.; GUTIÉRREZ, K.; GAXIOLA, J.; ARIAS, V.; BAUTISTA, M. Cambio en la cobertura y uso de suelo en el norte de Jalisco, México: Un análisis del futuro, en un contexto de cambio climático. Ambi-Agua, Taubaté, v. 6, n. 2, p. 111-128, 2011. (doi:10.4136/ambi-agua. 189)

\subsection{Predicción de uso de suelo y vegetación del año 2040 , en un contexto de cambio climático}

Con la aplicación del modelo para predecir el uso de suelo y vegetación del año 2040, se predice que el bosque de pino de la subcuenca Huichol-Atengo, estaría en riesgo de transformarse, principalmente en bosque de encino, este cambio estaría dado debido principalmente a la tolerancia que presentan las poblaciones de encinos a una disminución a la precipitación y al aumento de la temperatura.

Aunque, según el modelo desarrollado, los cambios en la temperatura no alcanzan los dos grados que señalan Villers y Trejo (2010), la disminución de la precipitación si alcanza los limites críticos para contribuir al cambio climático en la subcuenca Huichol-Atengo, por lo que se estima que la vegetación vulnerable es la que comprende el bosque de pino, por lo que se espera que el cambio se refleje en la abundancia de la vegetación de Quercus cambiando la relación Pino-encino por Encino-Pino y por ello se plantea una transición en la vegetación del tipo Pino-Encino para dar paso a la asociación Encino-Pino o en definitiva se transformará en bosques de encino.

Es posible que con estos cambios de temperatura y humedad otras comunidades vegetales de la subcuenca también enfrenten presiones, como por ejemplo, el incremento en la vegetación de pastizal, la cual se espera que se presente en tiempos relativamente cortos. Por otro lado, también, la capacidad de respuesta de las comunidades vegetales se vería afectada por el estado de conservación y por los factores como la deforestación.

\section{CONCLUSIÓN}

El estudio se caracterizó por presentar un nivel de análisis más detallado, delimitando el trabajo a nivel de subcuenca, con lo que fue posible estimar los cambios en la cobertura del terreno en función de las características particulares del área.

Por otro lado, se documenta la importancia del uso de los sistemas de información geográfica en el estudio de los cambios en la cobertura y uso de suelo de una determinada área.

Los modelos de cambio climático aplicados para predecir la cobertura vegetal y el uso de suelo de la subcuenca Huichol-Atengo para el año 2040, demostraron ser una metodología confiable para analizar los cambios en los tipos de vegetación hacia el futuro.

Por su parte, estos modelos resaltan los tipos de vegetación de la subcuenca HuicholAtengo para el año 2040, más sensibles a los cambios, tanto en el aumento de la temperatura como a la disminución en la precipitación y sientan las bases para iniciar una discusión acerca del tipo de medidas que sería conveniente aplicar, con el fin de mitigar los efectos del cambio climático en el uso de suelo y vegetación de la subcuenca Huichol-Atengo.

Los resultados de este trabajo ofrecen una posibilidad de utilizarlos en la planeación del territorio, a través de instrumentos como el ordenamiento ecológico territorial o los planes sectoriales de desarrollo como el urbano y el agrícola; o bien, en la definición de políticas de manejo de los recursos naturales y ecosistemas, aportando elementos descriptivos, cuantitativos y geográficos, que pueden apoyar la toma oportuna de decisiones a diferentes niveles de decisión y acción, y con ello contribuir a un desarrollo ecológico, social y económicamente sustentable en esta región. 


\section{AGRADECIMIENTOS}

Al Dr. Enrique Martínez-Meyer, por la revisión y sus comentarios que ayudaron para llevar a buen termino este trabajo. Al c. Dr. Humberto Cuellar, por su ayuda con la revisión del escrito; y al equipo de SAP, por su apoyo en los trabajos de campo.

\section{REFERENCIAS}

CHEN, L. Y.; YANG, H. C. Scenario simulation and forecast of land use/cover in northern China. Chinese Science Bulletin, v. 53, n. 9, p. 1401-1412, 2008.

http://dx.doi.org/10.1007/s11434-008-0169-9

DIRZO, R.; GARCÍA, M. C. Rates of deforestation in Los Tuxtlas a neotropical area in southeast Mexico. Conservation Biology, v. 6, n. 1, p. 84-90, 1991. http://dx.doi.org/10.1046/j.1523-1739.1992.610084.x

DURAN, P. Modelo de distribución de los pastizales en zonas áridas de México, ante los efectos del cambio climático. 2010. Tesis (Doctoral) - Universidad Nacional Autónoma de México, Coyacán, 2010.

FOOD AND AGRICULTURE ORGANIZATION OF THE UNITED NATIONS - FAO. Forest resource assessment. Disponible en: <http:www.fao.org>. Acesso en: 2005.

GARCÍA MORA, T. J.; MAS, J. F. Comparación de metodologías para el mapeo de la cobertura y uso del suelo en el sureste de México. Investigaciones Geográficas, n. 67, p. 7-19, 2008.

GONZÁlEZ, A.; BOJORQUEZ, J. I.; NÁJERA, O.; GARCÍA, J. D.; MADUEÑO, A.; FLORES, F. Regionalización ecológica de la llanura costera norte de Nayarit, México. Investigaciones Geográficas, n. 69, p. 21-32, 2009.

INSTITUTO NACIONAL DE ECOLOGIA - INE. Continuo nacional del conjunto de datos vectoriales de uso de suelo y vegetación, 1: 250 000, serie T3. México, DF: Secretaria del Medio Ambiente y Recursos Naturales (SEMARNAT), 2000.

LAMBIN, E. F. Modelling and monitoring land-cover change processes in tropical regions. Progress in Phisycal Geography, v. 21, n. 3, p. 375-393, 1997. http://dx.doi.org/10.1177/030913339702100303

MÁRQUEZ GONZÁLEZ, A. R. Cambio de uso de suelo y el desarrollo turístico en Bahía de Banderas, Nayarit. Ciencia UANL, v. 11, n. 2, p. 161-167, 2008.

MAS, J. F.; SORANI, V.; ÁLVAREZ, R. Elaboración de un modelo de simulación del proceso de deforestación. Investigaciones Geográficas, n. 5, p. 43-57, 1996.

MAS, J. F.; VELÁZQUEZ, A.; COUTURIER, S. La evaluación de los cambios de cobertura/uso del suelo en la República Mexicana. Investigación Ambiental, v. 1, n. 1, p. 23-39, 2009.

NÁJERA, G. O.; BOJORQUEZ, J. L.; VILCHEZ, F. F. Cobertura del terreno y uso del suelo de la reserva ecológica sierra de San Juan, Nayarit. En: REUNIÓN DE INVESTIGACIÓN Y DESARROLLO TECNOLÓGICO, 4., 2000, Tepic. Memorias... Tepic: [s.n], 2000. p. 180-181. 
OCHOA-GAONA, S.; GONZÁLEZ-ESPINOSA, M. Land use and deforestation in the highlands of Chiapas, Mexico. Applied Geography, v. 20, n. 1, p. 17-42, 2000. http://dx.doi.org/10.1016/S0143-6228(99)00017-X

REYES HERNÁNDEZ, H.; AGUILAR ROBLEDO, M.; AGUIRRE RIVERA, J. R.; TREJO VAZQUEZ, I. Cambios en la cobierta vegetal y uso de suelo en el área del proyecto Pujal-Coy, San Luís Potosí, México, 1973-2000. Investigaciones Geográficas, n. 59, p. 26-42, 2006.

ROSETE, F. A.; PÉREZ, J. L.; BOCCO, G. Contribución al análisis del cambio de uso del suelo y vegetación (1978-2000) en la Península de Baja California, México. Investigación Ambiental, n. 1, p. 70-82, 2009.

RZEDOWSY, J. Vegetación de México. 1. ed. digital. Comisión Nacional para el Conocimiento y Uso de la Biodiversidad, México, 2005. 504p. Disponible en: $<$ http://www.biodiversidad.gob.mx/publicaciones/librosDig/pdf/VegetacionMxPort.pdf >. Acesso: 10 mayo 2011.

MÉXICO. Secretaria del Medio Ambiente y Recursos Naturales - SEMARNAT. Informe de la Institución del medio ambiente en México. México, DF: SEMARNAT, 2005.

MÉXICO. Secretaria del Medio Ambiente y Recursos Naturales - SEMARNAT. La gestión ambiental en México. México, DF: SEMARNAT, 2006.

TREJO, I.; DIRZO, R. Deforestation of seasonally dry tropical forest: a national and local analysis in Mexico. Biological Conservation, v. 94, n. 2, p 133-142, 2000.

http://dx.doi.org/10.1016/S0006-3207(99)00188-3

TREJO, I.; HERNÁNDEZ, J. Identificación de selva baja caducifolia en el estado de Morelos, mediante imágenes de satélite. Investigaciones Geográficas, n. 5, p. 11-18, 1996.

TURNER, M. G. Landscape ecology: the effect of pattern on processes. Annual Review of Ecology and Systematics, n. 20, p. 171-197, 1989. http://dx.doi.org/10.1146/annurev.es.20.110189.001131

VELÁZQUEZ, A.; MAS, J. F.; PALACIO, J. L.; DÍAZ-GALLEGOS, J. R.; MAYAGORTASAUCEDO, R.; ALCÁNTARA, P. C. et al. Análisis de cambio de uso del suelo. Convenio [S.l.]: INE/IGg; UNAM, 2002.

VILLERS-RUÍZ, L.; TREJO-VÁZQUEZ, I. El cambio climático y la vegetación en México. 2010. Tesis (Doctoral) - Instituto de Geografía, Universidad Nacional Autónoma de México, México, DF, 2010. 